\title{
The crowded crossroad to angiogenesis in systemic sclerosis: where is the key to the problem?
}

\author{
Mirko Manetti ${ }^{*}$, Serena Guiducci ${ }^{2}$ and Marco Matucci-Cerinic ${ }^{2}$ \\ See related research by Hirigoyen et al., http://www.arthritis-research.com/content/17/1/332
}

\begin{abstract}
In systemic sclerosis (SSc), peripheral vasculopathy is characterized by a progressive and irreversible loss of capillaries following endothelial cell injury, due to defects in both vascular repair and expected increase in new vessel growth (angiogenesis). The discovery of key molecular targets may help to develop the most effective therapeutic strategy for the SSc-related vasculopathy. A pathway worth targeting in SSc may include vascular endothelial growth factor, 165b isoform, an endogenous angiogenesis inhibitor abnormally expressed and released by different cell types, including activated endothelial cells and platelets.
\end{abstract}

Dysregulated expression of several proangiogenic and antiangiogenic factors has been implicated in the dysfunction of angiogenesis in systemic sclerosis (SSc). Into this complex scenario comes the study recently published in Arthritis Research \& Therapy by Hirigoyen et al. [1], who report new data implicating an inhibitory splice variant of vascular endothelial growth factor (VEGF)-A, namely $V_{E G F}{ }_{165} \mathrm{~b}$, in the inhibition of angiogenesis by platelets in SSc. These findings are part of an intriguing chain of data as several studies have shown that VEGF-A, one of the most potent promoters of angiogenesis, is markedly increased in SSc skin and circulation despite clear evidence of an insufficient angiogenic response [2, 3]. This was considered the "SSc paradox" until it became evident recently that early studies could not distinguish between proangiogenic $\mathrm{VEGF}_{165}$ and antiangiogenic $\mathrm{VEGF}_{165} \mathrm{~b}$ isoforms generated

\footnotetext{
* Correspondence: mirkomanetti@yahoo.it

${ }^{1}$ Department of Experimental and Clinical Medicine, Section of Anatomy and Histology, University of Florence, 50134 Florence, Italy
}

Full list of author information is available at the end of the article by alternative splicing in VEGF-A pre-mRNA [4]. In most pathologic angiogenic conditions, such as cancers, VEGF $_{165}$ predominates. On the contrary, this crucial balance between isoforms shifts in favor of the expression of $\mathrm{VEGF}_{165} \mathrm{~b}$ in SSc (Fig. 1) [4, 5]. $\mathrm{VEGF}_{165} \mathrm{~b}$ appears selectively overexpressed in different cell types of SSc dermis, including endothelial cells (ECs), fibroblasts, and perivascular mononuclear inflammatory cells [5]. In vitro studies further revealed that SSc dermal microvascular endothelial cells (MVECs) express and release elevated levels of $\mathrm{VEGF}_{165} \mathrm{~b}$ [5]. Moreover, the increased $\mathrm{VEGF}_{165} \mathrm{~b}$ plasma levels in SSc correlate with the extent of nailfold capillary architectural derangement and loss, suggesting their potential utility as a biomarker reflecting disease severity [6]. In this context, the study by Hirigoyen et al. [1] adds new valuable information showing that, in SSc, platelets store high levels of VEGF 165 b. It could therefore be suggested that platelets may be a major source of circulating $\mathrm{VEGF}_{165} \mathrm{~b}$ in SSc, in particular following their activation on contact with the injured endothelium.

The discovery of $V_{E G F}$ b5 clearly adds a new fundamental figure in the complex crossroad of the mechanisms underlying dysfunctional angiogenesis in SSc. Binding of VEGF 165 to vascular endothelial growth factor receptor (VEGFR)-2 induces VEGFR-2 internalization and activates signaling pathways that trigger EC proliferation, survival, and migration which are necessary for angiogenesis. VEGF ${ }_{165} \mathrm{~b}$ binds to VEGFR-2 with the same affinity as VEGF 165 , but binding of VEGF 165 b results in an insufficient tyrosine phosphorylation/activation of VEGFR-2 and incomplete downstream signaling (Fig. 1) [4]. Owing to increased levels of $V_{E G F}{ }_{165} \mathrm{~b}$, SSc-MVECs show reduced VEGFR-2 phosphorylation and impaired capillary morphogenesis in vitro [5]. Accordingly, the angiogenic performance of SSc-MVECs can be improved by cotreatment with recombinant $\mathrm{VEGF}_{165}$ and anti-VEGF ${ }_{165} \mathrm{~b}$ 


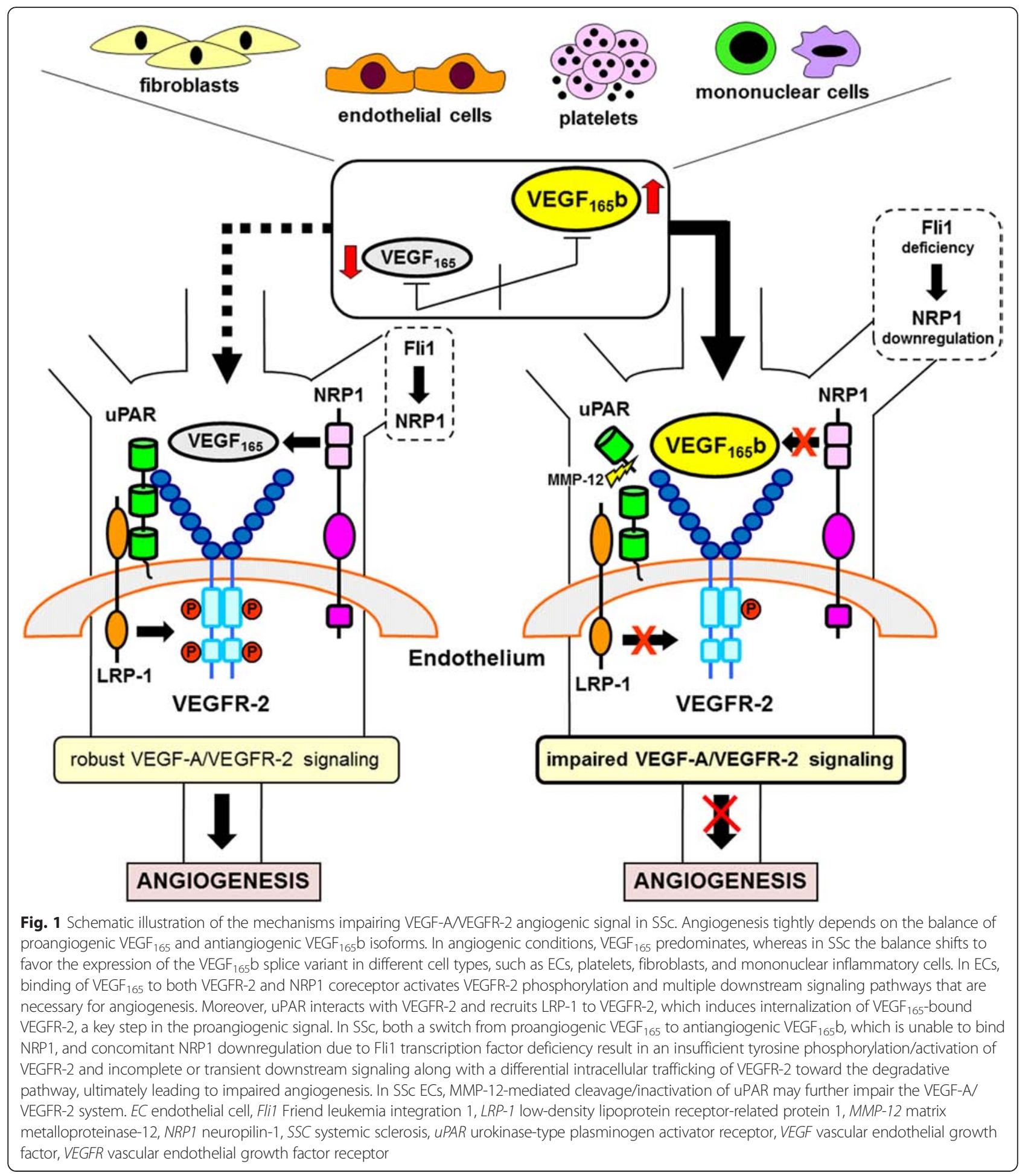

neutralizing antibodies [5]. Of note in the study by Hirigoyen et al. [1], when healthy dermal MVECs were incubated with SSc platelet releasates containing elevated levels of $\mathrm{VEGF}_{165} \mathrm{~b}$, capillary-like tube formation was significantly inhibited compared with cells treated with control platelet releasates.
It is known that the nontyrosine kinase receptor neuropilin-1 (NRP1) acts as a VEGFR-2 coreceptor in angiogenesis [7]. $\mathrm{VEGF}_{165} \mathrm{~b}$ antiangiogenic function is thought to be mechanistically related to the lack of NRP1 coreceptor binding [4]. In fact, upon binding of VEGF $_{165} \mathrm{~b}$ to VEGFR-2, the absence of NRP1 cosignaling 
induces differential trafficking of VEGFR-2 toward proteasomal degradation [4]. In SSc, besides VEGF ${ }_{165}$ b overexpression, concomitant NRP1 downregulation because of Friend leukemia integration 1 (Fli1) transcription factor deficiency may further disturb VEGF-A/VEGFR-2 signaling [8]. Finally, in this complex scenario the urokinase-type plasminogen activator receptor (UPAR), an angiogenic orchestrator acting via both VEGF-Adependent and VEGF-A-independent mechanisms, may play a significant role because UPAR inactivation represents a master factor challenging angiogenesis in SSc $[2,9]$. uPAR-VEGFR-2 interaction is crucial for VEGFR-2 internalization and proangiogenic signaling in ECs [10]. Of note, VEGF-A-induced angiogenesis is prevented in uPAR-deficient mice, a recently proposed animal model of SSc $[9,10]$. uPAR inactivation/deficiency may thus contribute substantially to VEGF-A/VEGFR-2 system abnormalities in SSc (Fig. 1).

Taken together, considerable evidence suggests that uncontrolled expression of the antiangiogenic VEGF $_{165} \mathrm{~b}$ isoform may take center stage in this crowded crossroad to angiogenesis in SSc. It remains to be elucidated whether the targeting of $\mathrm{VEGF}_{165} \mathrm{~b}$ and/or VEGF-A premRNA splicing machinery might represent a promising therapeutic strategy to promote effective angiogenesis in SSc patients. As pointed out in the article by Hirigoyen et al. [1], a better understanding of how platelets transport and deliver uncontrolled levels of $\mathrm{VEGF}_{165} \mathrm{~b}$ to the injured endothelium might disclose a novel pathway contributing to the SSc-related peripheral vasculopathy. Whether this mechanism is a priority pathway worth targeting warrants further studies.

\section{Abbreviations}

EC: endothelial cell; Fli1: friend leukemia integration 1; MVEC: microvascular endothelial cell; NRP1: neuropilin-1; SSc: systemic sclerosis; UPAR: urokinasetype plasminogen activator receptor; VEGF: vascular endothelial growth factor; VEGFR: vascular endothelial growth factor receptor.

\section{Competing interests}

The authors declare that they have no competing interests.

\section{Authors' contributions}

MM, SG, and MM-C contributed to the writing and revising of the manuscript and approved the final manuscript.

\section{Author details}

'Department of Experimental and Clinical Medicine, Section of Anatomy and Histology, University of Florence, 50134 Florence, Italy. ${ }^{2}$ Department of Experimental and Clinical Medicine, Division of Rheumatology, AOUC, University of Florence, 50139 Florence, Italy.

\section{(W)}

\section{References}

1. Hirigoyen D, Burgos PI, Mezzano V, Duran J, Barrientos M, Saez CG, et al. Inhibition of angiogenesis by platelets in systemic sclerosis patients. Arthritis Res Ther. 2015;17:332.

2. Manetti M, Guiducci S, Ibba-Manneschi L, Matucci-Cerinic M. Mechanisms in the loss of capillaries in systemic sclerosis: angiogenesis versus vasculogenesis. J Cell Mol Med. 2010;14:1241-54.
3. Distler O, Distler JH, Scheid A, Acker T, Hirth A, Rethage J, et al. Uncontrolled expression of vascular endothelial growth factor and its receptors leads to insufficient skin angiogenesis in patients with systemic sclerosis. Circ Res. 2004:95:109-16.

4. Manetti M, Guiducci S, Ibba-Manneschi L, Matucci-Cerinic M. Impaired angiogenesis in systemic sclerosis: the emerging role of the antiangiogenic VEGF(165)b splice variant. Trends Cardiovasc Med. 2011;21:204-10.

5. Manetti M, Guiducci S, Romano E, Ceccarelli C, Bellando-Randone S, Conforti ML, et al. Overexpression of VEGF165b, an inhibitory splice variant of vascular endothelial growth factor, leads to insufficient angiogenesis in patients with systemic sclerosis. Circ Res. 2011;109:e14-26.

6. Manetti M, Guiducci S, Romano E, Bellando-Randone S, Lepri G, Bruni C, et al. Increased plasma levels of the VEGF165b splice variant are associated with the severity of nailfold capillary loss in systemic sclerosis. Ann Rheum Dis. 2013:72:1425-7.

7. Lampropoulou A, Ruhrberg C. Neuropilin regulation of angiogenesis. Biochem Soc Trans. 2014:42:1623-8.

8. Romano E, Chora I, Manetti M, Mazzotta C, Rosa I, Bellando-Randone S, et al. Decreased expression of neuropilin-1 as a novel key factor contributing to peripheral microvasculopathy and defective angiogenesis in systemic sclerosis. Ann Rheum Dis. 2015. doi:10.1136/annrheumdis-2015-207483.

9. Manetti M, Rosa I, Milia AF, Guiducci S, Carmeliet P, Ibba-Manneschi L, et al. Inactivation of urokinase-type plasminogen activator receptor (UPAR) gene induces dermal and pulmonary fibrosis and peripheral microvasculopathy in mice: a new model of experimental scleroderma? Ann Rheum Dis. 2014;73:1700-9.

10. Herkenne S, Paques C, Nivelles O, Lion M, Bajou K, Pollenus T, et al. The interaction of UPAR with VEGFR2 promotes VEGF-induced angiogenesis. Sci Signal. 2015;8:ra117. 